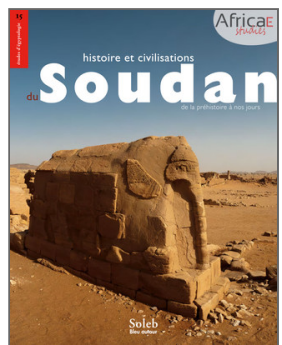

Olivier Cabon (dir.)

Histoire et civilisation du Soudan

De la préhistoire à nos jours

\title{
Regards sur Khartoum
}

\section{Odile Nicoloso et Nicolas Beaumé}

DOI : 10.4000/books.africae. 2952

Éditeur : Africae, Soleb, Bleu autour

Lieu d'édition : Paris, Khartoum

Année d'édition : 2017

Date de mise en ligne : 17 janvier 2022

Collection : Africae Studies

EAN électronique : 9782493207074

\section{OpenEdition}

\section{Books}

http://books.openedition.org

\section{Référence électronique}

NICOLOSO, Odile ; BEAUMÉ, Nicolas. Regards sur Khartoum In : Histoire et civilisation du Soudan : De la préhistoire à nos jours [en ligne]. Paris, Khartoum : Africae, 2017 (généré le 28 janvier 2022). Disponible sur Internet : <http://books.openedition.org/africae/2952>. ISBN : 9782493207074. DOI : https:// doi.org/10.4000/books.africae.2952. 

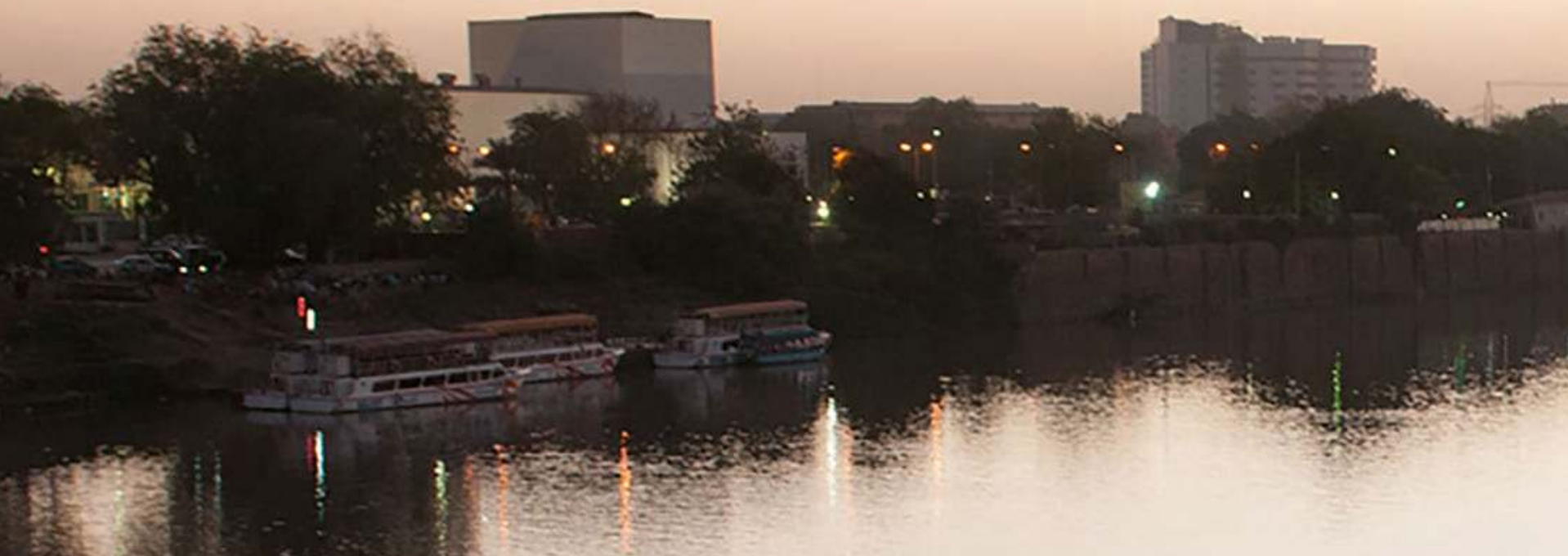


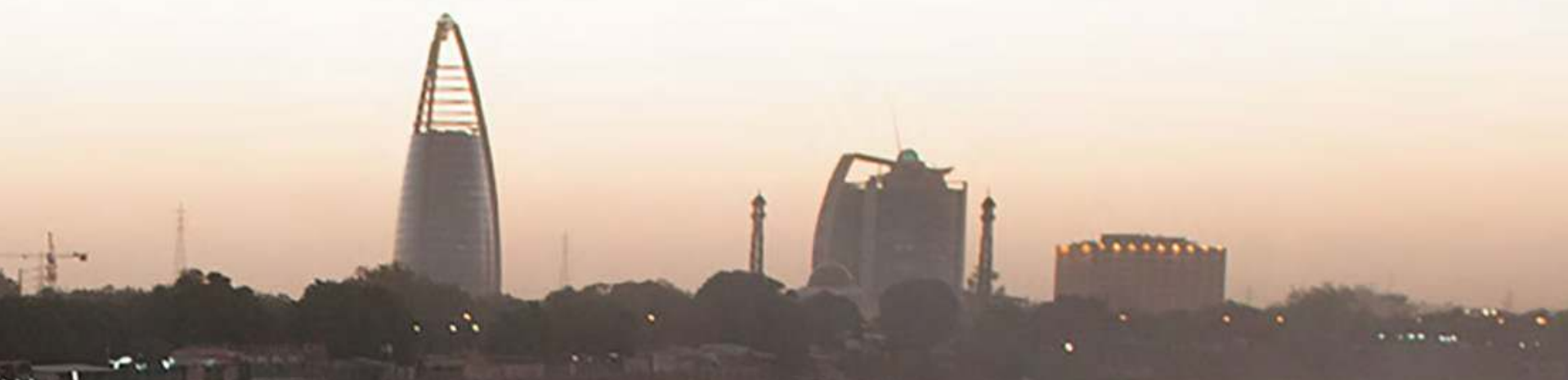

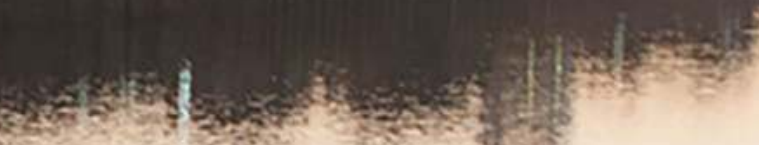
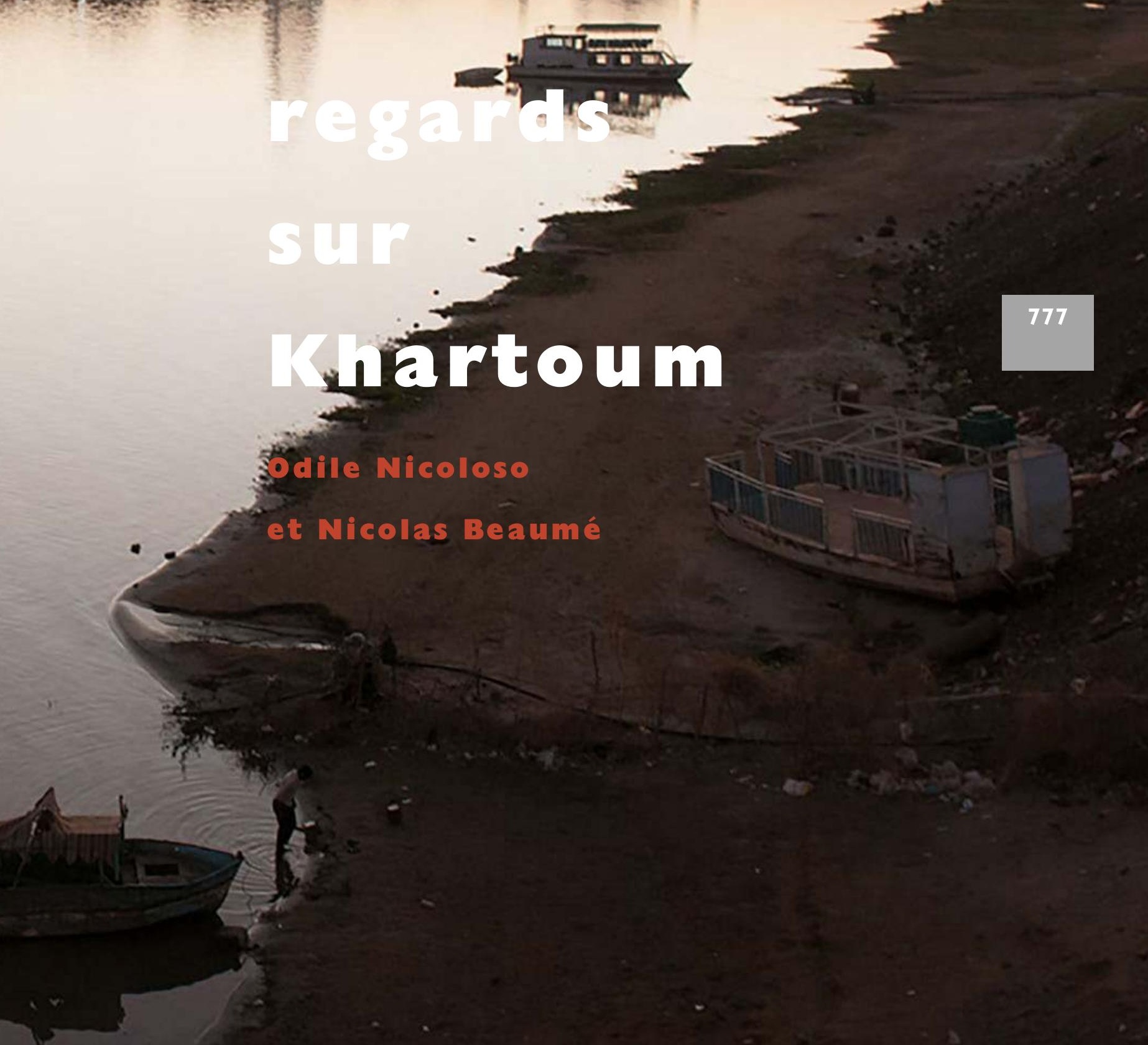


\section{regards sur Khartoum}

Khartoum en 1924

souvenirs de la famille Kafouri

En 1924, Khartoum n'était plus la cité rose de Gordon. La plupart des maisons étaient en brique et zibala ou banco (mélange de boue, de fumier de cheval ou de vache). Les rues étaient larges et désertes, il était fréquent que les rares voitures s'ensablent; les ânes qui étaient alors le principal moyen de locomotion, créaient de fréquents embouteillages avec les chameaux, à l'entrée de l'unique pont sur le Nil où un péage était requis. Dans les réceptions officielles, les âniers rivalisaient afin d'avoir le plus bel âne et le plus beau harnachement. La plupart des maisons avaient leur propre puits d'eau potable. Lélectricité étant réduite au minimum, les rues étaient plongées dans l'obscurité. Il n'y avait pas de réfrigérateurs, seulement des glacières que fabriquait le menuisier. Les jours de grande chaleur, tous se précipitaient à la fabrique de glace, mais ils revenaient souvent bredouilles! Il n'y avait bien sûr ni radio, ni télévision, ni journaux. Le courrier arrivait deux fois par semaine. La charrette des latrines, tirée par deux chameaux, arrivait le soir, généralement au moment du dîner, provoquant l'irritation de tous. Les nuits étaient difficiles, la chaleur empêchait de dormir, ainsi que, à l'intérieur, le bruit agaçant du ventilateur en bois et, à l'extérieur, des multitudes de moustiques. Il était pratiquement impossible d'allumer une lumière car elle aurait provoqué une invasion d'insectes divers et variés qui mordaient, volaient, bourdonnaient, rampaient. La nourriture arrivait couverte d'un let que vous deviez remettre rapidement pour recouvrir le plat après vous être servi.

Malgré ces désagréments, la chaleur, les moustiques et la malaria, la bonne société organisait ses loisirs : conversations en bonne compagnie, lectures, écoute de la musique sur un gramophone et pratique de quelques sports, tels que le tennis, le golf, le cricket, le football, l'équitation...

Puis le téléphone et l'automobile arrivèrent; en 1924, on comptait 200 voitures dans tout le Soudan. Un Copte créa un cinéma en plein air qui devint le but de sortie de nombreux jeunes de Khartoum.

\section{Le quartier Kafouri à Khartoum entre 1948 et 1970 rencontre avec Charles Kafouri}

Aziz Kafouri, le père de Charles et Alex, né en 1874, venait de Dhour el Chouer, dans les montagnes du Liban, où sa famille possédait une filature de soie. De religion grecque orthodoxe, il fit ses études dans une école franco libanaise à Antura. Le commerce de la soie s'étant effondré et son père étant décédé, il dut partir reconstruire la fortune familiale, à 
Alexandrie, puis à Khartoum à partir de 1899 . Il arriva par le train, mais comme celui-ci ne parvenait pas encore jusqu’à Khartoum, il fit certainement les derniers kilomètres en camion. Il contribua à reconstruire la ville détruite pendant la révolte mahdiste, grâce notamment au commerce de bois et matériaux de construction; il devint ainsi par la suite un des premiers hommes d'affaires du Soudan avec quelques Grecs et Italiens.

Son premier fils naquit en 1900 à Omdurman où il vécut à son arrivée. Quatre autres fils suivirent, nés dans la maison qui devait devenir plus tard l'hôtel Saint-James. La famille comptera au final sept garçons qu'Aziz installa en 1920 sur la rive nord du Nil où il acquit les terrains qui devaient devenir le quartier Kafouri. La prison Kober existait déjà — construite par les Britanniques, elle doit son nom, qui fut ensuite quelque peu déformé, à son premier directeur qui s'appelait Cooper. En 1952, les deux plus jeunes fils, Charles et Alex, construisirent une maison plus moderne qu'ils habitèrent jusqu'en 1997.

Les Kafouri furent les pionniers du système d'irrigation par pompes. Ce fut une tâche ardue à cause de la teneur en sel des terres. Après quelques années de drainages et grâce à la construction de nouvelles canalisations, ils réussirent à produire du sorgho de bonne qualité et, surtout, du coton, ce jusqu’à la crise financière mondiale de 1929 à la suite de laquelle Aziz dut alors vendre une partie de ses biens.

Une ferme laitière d'environ mille feddans gérée par les Britanniques, située à côté de la nonciature actuelle, fournissait le lait à la communauté britannique grâce à des vaches croisées importées d'Angleterre. Le gouverneur britannique de l'époque, qui voulait se décharger de cette affaire, proposa à Aziz de l'acquérir en 1932. Ainsi le cinquième fils, Gabriel, fut-il nommé directeur de cette laiterie. Bien que n'ayant aucune compétence au départ en ce domaine, il réussit à faire de Belgravia Dairy une institution de Khartoum. Passée de 70 têtes de bétail en 1932 à 300 en 1940, elle comptera en 1960 jusqu’à 2000 têtes de bétail. Le lait était livré chaque matin, à crédit, à domicile: au début par des charrettes tirées par des chevaux, à partir de 1934 par des camionnettes.

Pendant la Seconde Guerre mondiale, Gaby Kafouri put ainsi ravitailler les militaires en poste ou en transit vers la frontière éthiopienne et fournir le fourrage pour leurs chevaux.

Ses autres frères vaquant à d'autres activités et Gaby s'étant retiré en Angleterre en 1952, Charles, le sixième fils, devint directeur de toutes les activités liées à la ferme et à la laiterie. Il diversifia la société avec l'aide d'Alex, en lançant notamment la fabrication de glaces. Dans les années 1949-1970 qui virent six régimes politiques se succéder au Soudan les deux frères développèrent les anciennes activités familiales, comme 
le Soudan

780

aujourd'hui fourniture de bois et matériaux de construction, puis, dans les années soixante, créèrent une imprimerie, une usine de peinture, une autre de tabac et investirent dans la publicité, ceci jusqu'en 1970, date des nationalisations et confiscations.

À partir des années cinquante, le quartier, d'une superficie de 1300 hectares, commença à s'urbaniser. En 1970, les confiscations furent un coup très rude pour la famille Kafouri qui dut quitter le Soudan. Les autorités s'engagèrent à payer des compensations sur cinq ans mais seule une partie fut effectivement versée. Après différents accords avec le gouvernement Neimery, Charles continua à s'occuper de la société et à maintenir le nom Aziz Kfouri, avec l'aide d'Alex, qui s'était engagé dans une carrière académique mais revenait deux fois par an.

Dans les années quatre-vingt, la ferme laitière, qui ne pouvait plus rester en centre-ville, cessa toute activité et, avec son bétail, fut rachetée par le gouvernement. La zone, alors déclarée constructible, devint la "cité Kafouri», dont environ $10 \%$ appartenaient aux actionnaires de la société Aziz Kfouri; des projets d'immeubles, de rues, de services publics, d'écoles, de jardins d'enfants, de commerces furent réalisés.

En 2005 fut créée la Kfouri Technical Company, spécialisée dans les produits dérivés du pétrole.

Aujourd'hui, Norma Kafouri (Charles est décédé en 2015) habite toujours la maison familiale semblable à un bateau, sur les bords du Nil, dans ce quartier qui porte son nom. Elle est impliquée, elle aussi, dans la vie soudanaise, particulièrement dans diverses associations caritatives comme l'International Women Group (IwG) dont les dons vont à diverses écoles, hôpitaux ou autres œuvres sociales.

\section{L'Acropole}

Cet hôtel mythique de Khartoum - et un des plus anciens - est situé dans le vieux centre-ville. Il fut construit en 1952 par la famille grecque Pagoulatos. Panaghis, le père, à la recherche d'un avenir meilleur, était venu de Céphalonie en 1936. Après avoir un temps fait du commerce en Éthiopie avec son frère aîné, il décida de créer cet hôtel. Il décéda prématurément en 1967. Son épouse, Flora, Grecque d'Égypte parlant cinq langues, a élevé ses trois garçons tout en continuant à gérer l'hôtel. Elle en fut le pilier et inculqua le sens du travail et de la discipline à ses fils qui, aujourd'hui, se partagent le travail, chacun dans son domaine privilégié. C'est une entreprise familiale qui a accompagné la vie culturelle et politique du Soudan et a donc dû s'adapter aux différents aléas de son histoire. À son époque glorieuse, si on peut s'exprimer ainsi, l'Acropole 
possédait également trois boutiques de spiritueux et une pâtisserie de type européen, ainsi qu'un restaurant en terrasse qui dominait tout Khartoum et où étaient projetés des films une fois par semaine.

Les premiers clients de l'hôtel furent d'abord des ONG comme Oxfam puis MsF, particulièrement présents lors de la guerre en Érythrée et durant la période de sécheresse et de famine en 1984-1985. Ses fax, télex et téléphones étant les meilleurs de Khartoum, l'Acropole devint leur quartier général puis le point de ralliement des archéologues, journalistes, professeurs, écrivains de toutes nationalités, hommes d'affaires et même des spécialistes du renseignement. L'Acropole était une plaque tournante, un lieu de rencontre où auraient pu se côtoyer Bob Geldorf, le terroriste Carlos ou Oussama Ben Laden, qui à ce moment-là n'était qu'un riche homme d'affaires saoudien.

Deux Américains y organisèrent le retour des Falachas, juifs d'Éthiopie, vers Israël, avant d'être arrêtés par la police soudanaise. Malheureusement, l'hôtel fut le théâtre en 1988, d'un attentat organisé par les Palestiniens qui coûta la vie à sept personnes dont deux enfants, détruisit le bâtiment principal et interrompit pendant deux ans la vie de l'établissement. À la promulgation de la loi islamique, en 1983, les commerces annexes furent fermés. Depuis, l'hôtel a revu ses normes de sécurité et les clients sont revenus. Les Pagoulatos se sont spécialisés dans le tourisme, le catering. Georges et ses frères ont la réputation d'être les meilleures "personnes-ressources» pour organiser un voyage au Soudan. Ils accueillent hommes d'affaires, touristes, consultants, archéologues... L'hôtel garde son charme désuet - si bien décrit par Olivier Rolin dans son roman Méroé - et l'accueil familial donne à chaque client le sentiment d'être «à la maison».

\section{l'île de Tuti et les trois Nils}

L'île de Tuti est un village agricole au milieu de la ville. D'une surface de 42 hectares, elle héberge 15000 habitants. Jadis elle fournissait tout Khartoum en légumes. À la pointe nord de l'île, le Nil Bleu, venant du sud-est, et le Nil Blanc, de l'ouest, se rejoignent pour former le grand Nil. Un pont suspendu d'une largeur de 20 mètres inauguré, en mars 2009, la relie désormais au centre urbain. Auparavant, les habitants de l'île traversaient plusieurs fois par jour le fleuve en ferry pour rejoindre la terre ferme; cet isolement a créé un fort sentiment d'appartenance et de solidarité. Ce pont - et le projet de deux autres supplémentaires - leur apporte, certes, la modernité... mais leur fait craindre la spéculation immobilière qui bousculerait leur mode de vie traditionnel et les pousserait malgré eux dans le $\mathrm{XXI}^{\mathrm{e}}$ siècle. 


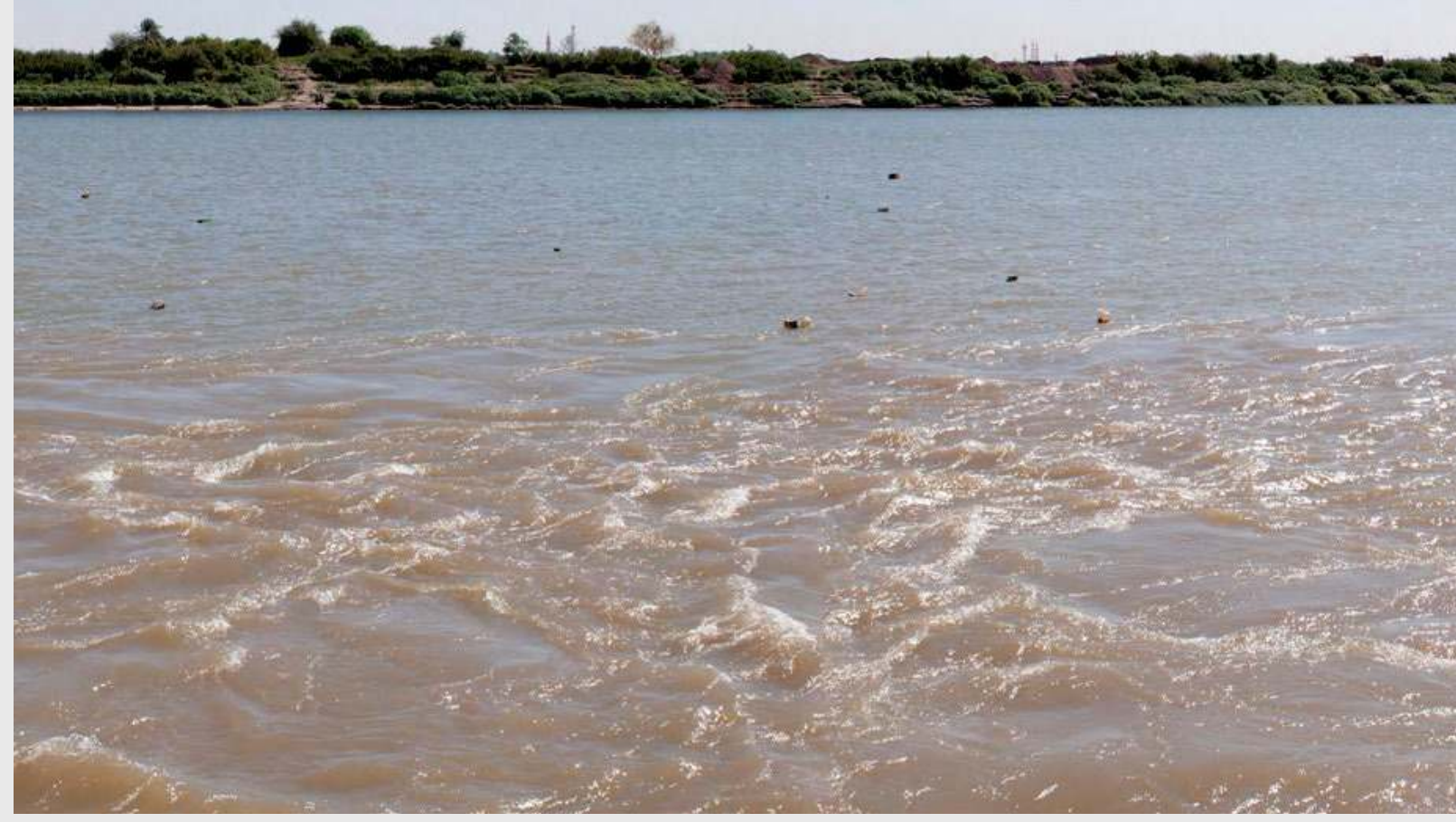

Pointe de l'île de Tuti : la confluence des deux Nils, al-Mogran, est un des hauts lieux géographiques de l'Afrique: le Nil Bleu (à gauche, venant
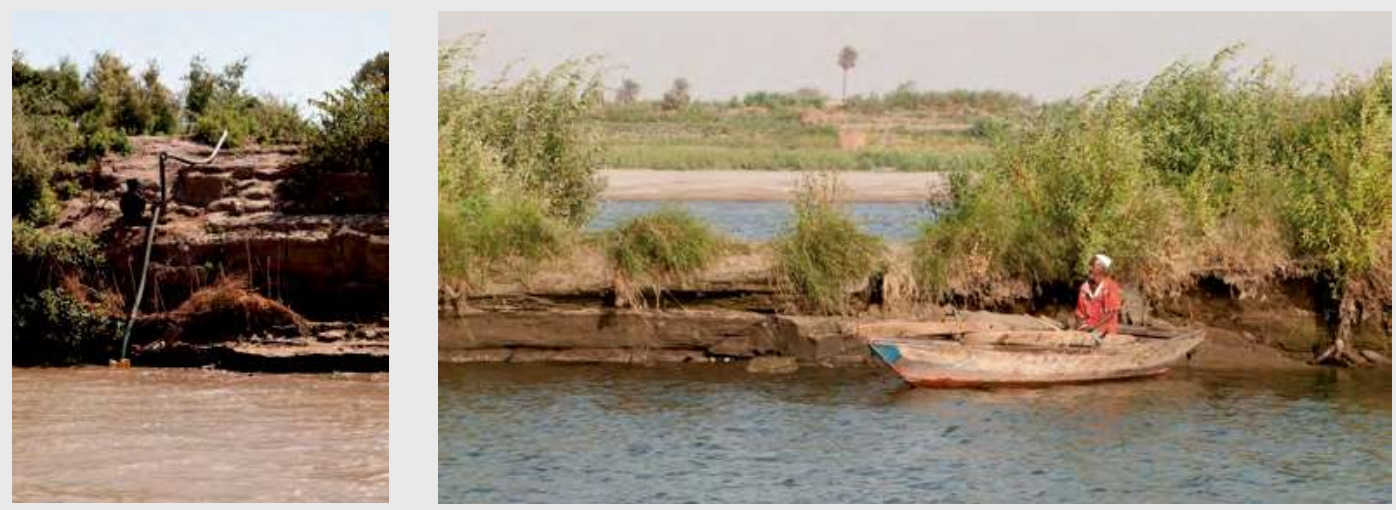

L'île de Tuti, d'une surface de 42 hectares et peuplée de 15000 habitants, fournissait jadis tout Khartoum en légumes. Au sud, un pont suspendu 


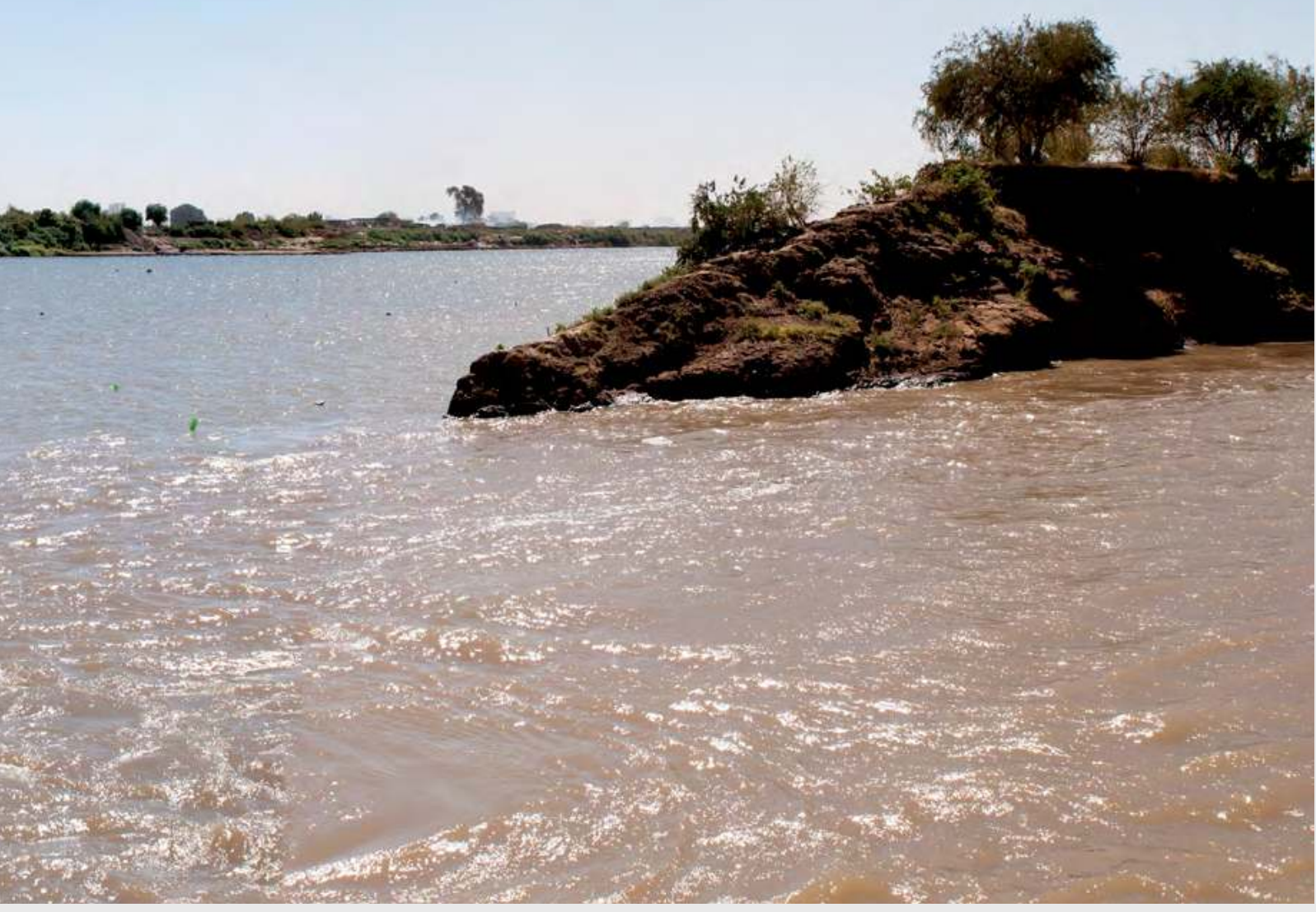

d'Éthiopie), rapide et étroit, se mélange avec le Nil Blanc (à droite, arrivant d’Ouganda), large et paresseux après sa traversée du lac Victoria et des marais.

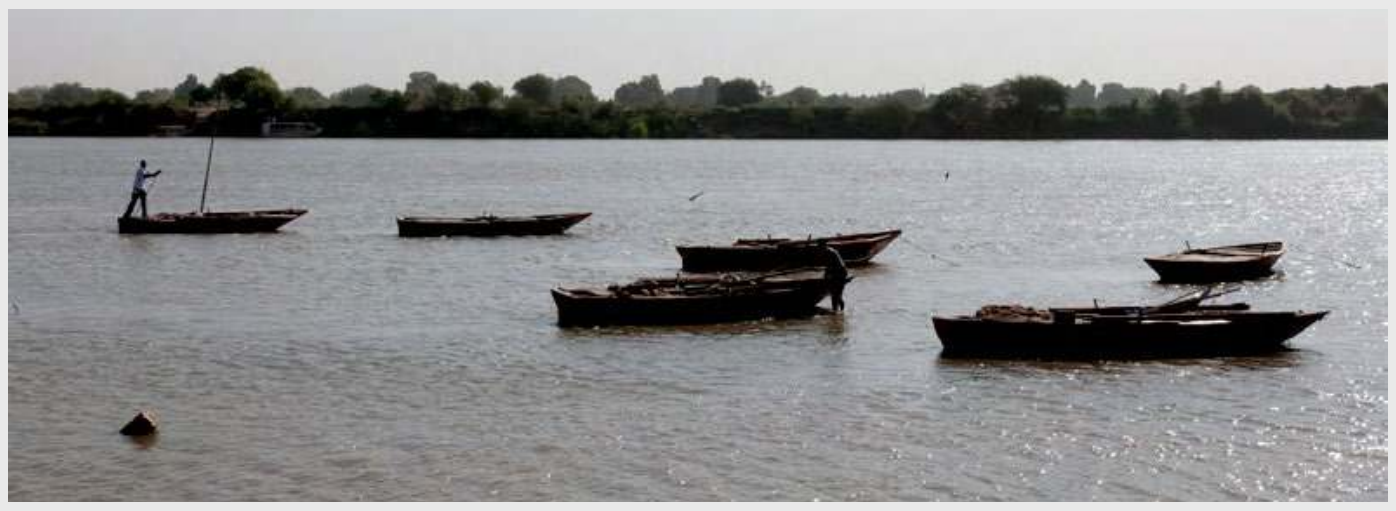

d'une largeur de 20 mètres la relie désormais au centre urbain. (À sa pointe nord, le Nil Bleu et le Nil Blanc se rejoignent pour former le grand Nil.) 


\section{Khartoum en 2012}

Les deux Nils - le Bleu et le Blanc — se rencontrent à Khartoum pour ne constituer qu'un seul fleuve qui remonte jusqu’à la Méditerranée. La confluence des deux Nils, nommée al-Mogran, est un des hauts lieux géographiques de l'Afrique. De cet endroit, vous pouvez observer le Nil Bleu, rapide et étroit, venir d'Éthiopie puis se mélanger avec le Nil Blanc, large et paresseux après sa traversée du lac Victoria et des marais du sud. Pendant les mois d'été, quand le Nil Bleu est à son plus haut niveau, sa puissance est telle qu'il oblige le Nil Blanc à refluer et inonde parfois les quartiers sud. À ce moment-là leur différence de couleur est très visible.

La ville s'est imposée comme capitale depuis l'époque du condominium; elle est le melting-pot où se rencontrent la plupart des groupes ethniques qui forment le Soudan. Elle est constituée en quelque sorte de trois entités: Khartoum, Khartoum Nord et Omdurman, la vieille ville, nommée ville sainte par le Mahdi où il s'établit et où se trouve son tombeau. Omdurman, aux maisons en brique crue et au souk immense, est la ville populaire et arabe. Plusieurs générations se partagent encore la même maison et vivent de manière collective et très simple. Grands-parents, enfants et petits-enfants logent sous le même toit, dormant souvent dehors à la belle étoile - l'intimité est l'apanage des classes sociales aisées qui ont acquis des habitudes occidentales ou qui vivent dans les grandes villes. La construction de tours qui abritent des appartements et de compounds résidentiels de luxe remet en question l'ancien habitat traditionnel. Quant au vieux centre-ville de Khartoum, il possède encore quelques bâtiments coloniaux au charme suranné où les églises côtoient les mosquées.

Le souk d'Omdurman n'est pas un souk très ancien, il n'a pas le charme de ceux du Caire ou d'Istamboul, mais il est très vaste et déborde d'activité.

Khartoum est le centre politique et économique du pays. Après la seconde guerre civile, un véritable boom a enfiévré Khartoum avec la manne pétrolière, la globalisation et l'arrivée de l'ONU et des ONG qui offraient du travail. De nouvelles routes et de nouvelles tours inspirées par Dubaï ont surgi du sol, des écrans géants ont fait leur apparition aux carrefours, de nombreux parcs d'attractions ont vu le jour et attirent les familles le week-end. À la périphérie, cependant, vit tout un petit peuple de réfugiés, Sud-Soudanais, Darfouris ou étrangers, petits commerçants, ou paysans de la Gezira, musulmans ou chrétiens, qui s'agglutinent dans des maisons en terre, sans eau courante ni électricité. Khartoum compte quatre camps officiels de déplacés: Mayo, es-Salam, Gébel Aulia, Wad el-Bechir 


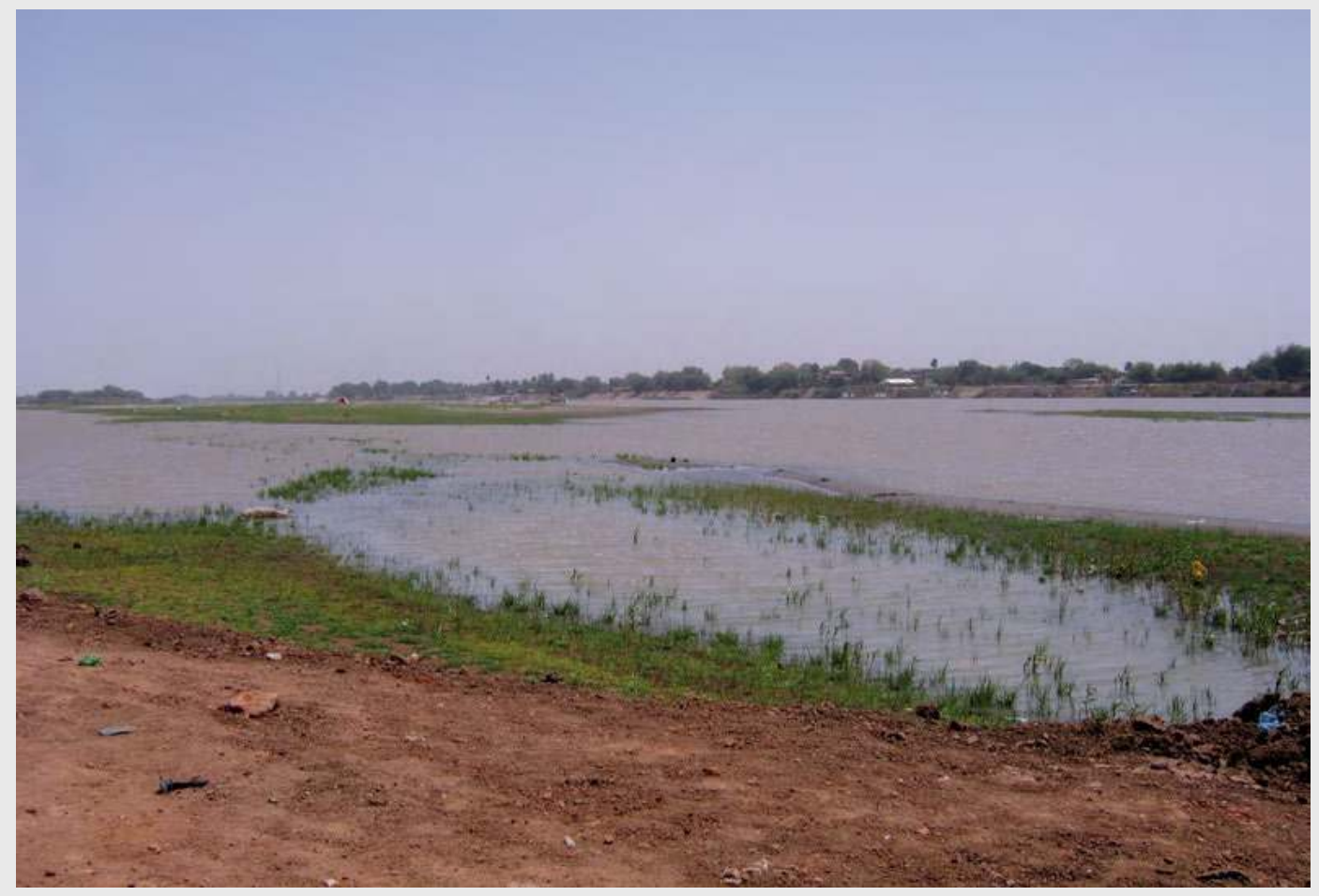

Crue du Nil, plus importante que d'habitude et inondant les champs, à Khartoum, en 2011.

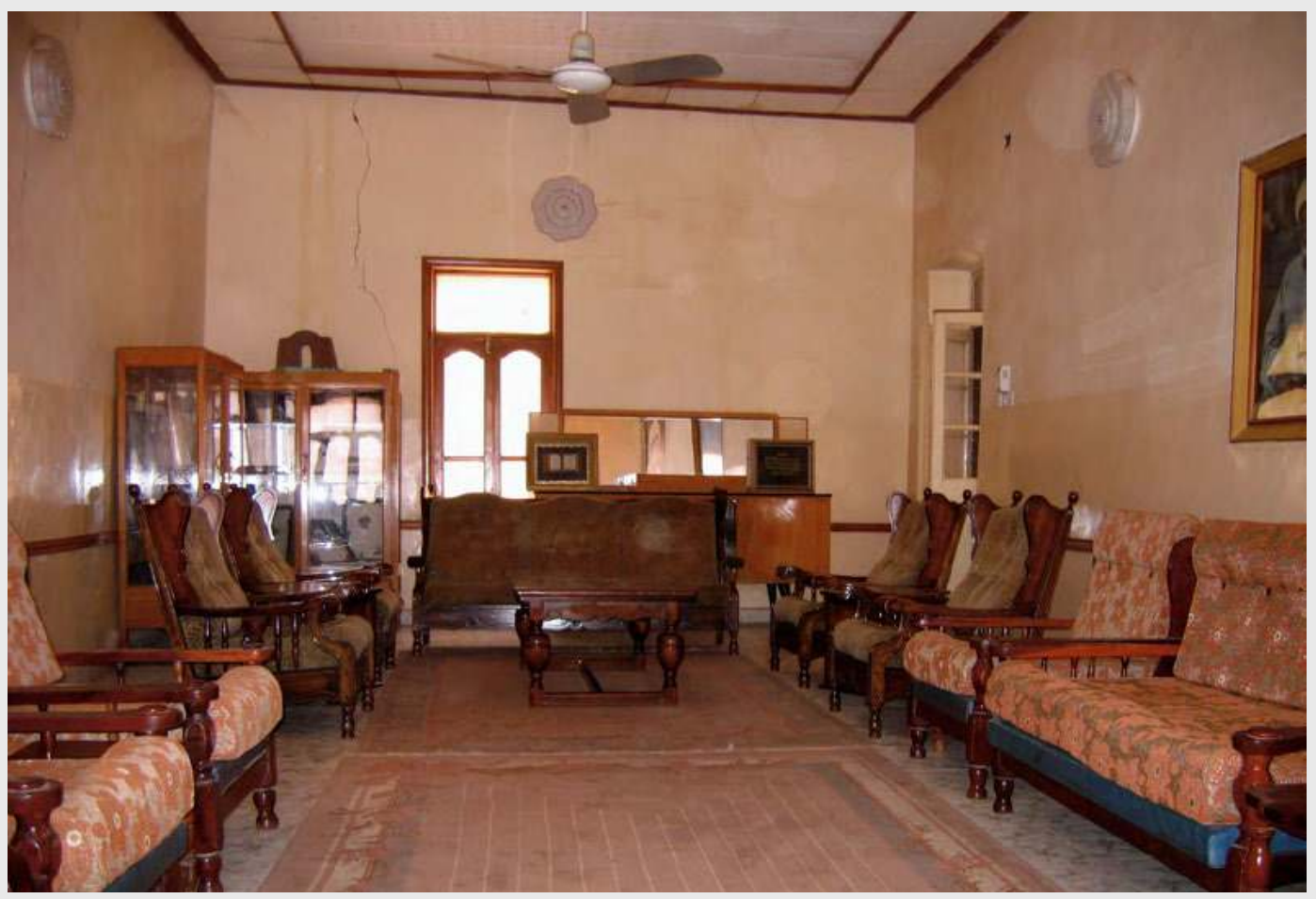




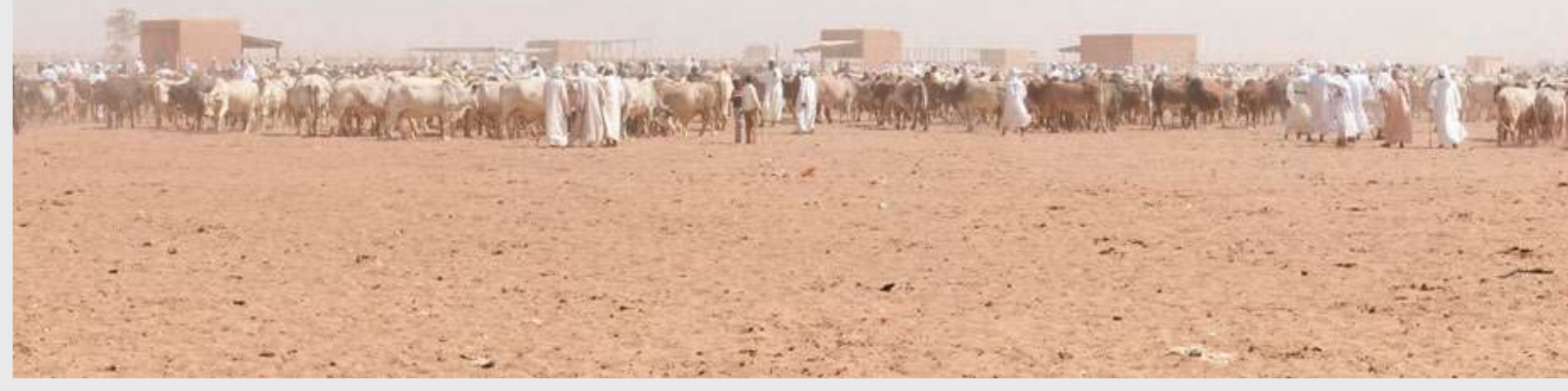

le Soudan

\section{6}

aujourd'hui
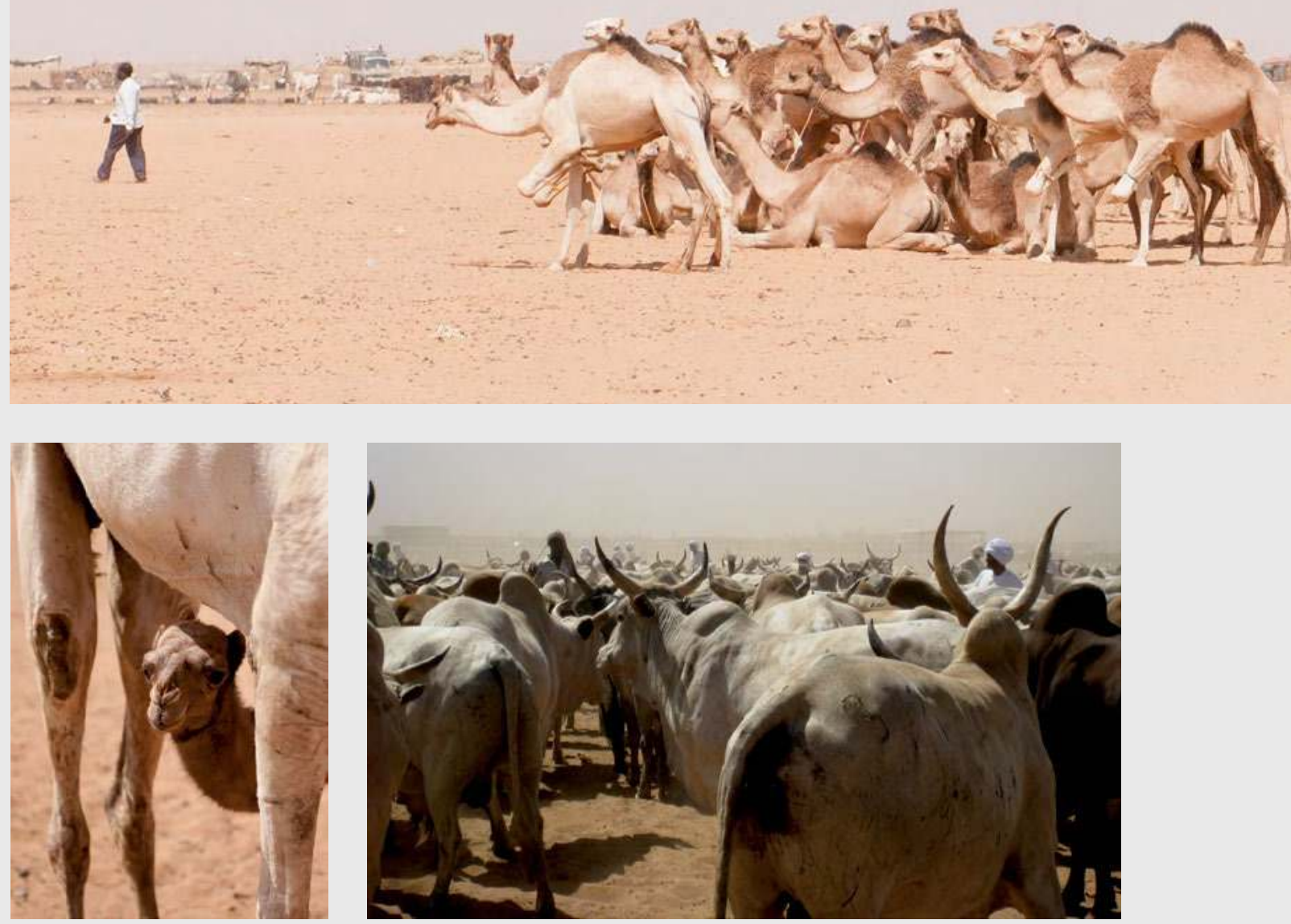

Les marchés aux chameaux et aux booufs et la maison du Mahdi à Omdurman. 

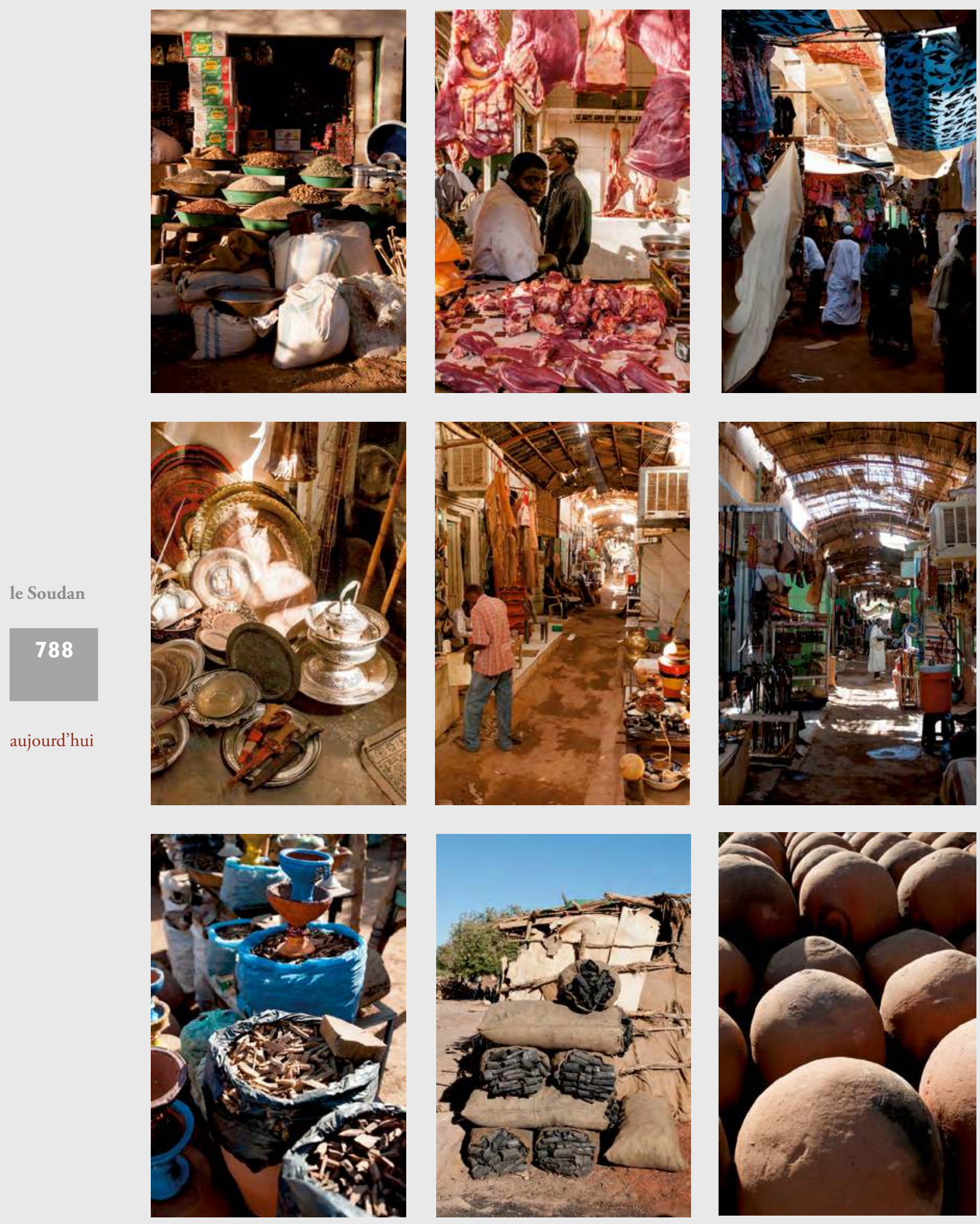

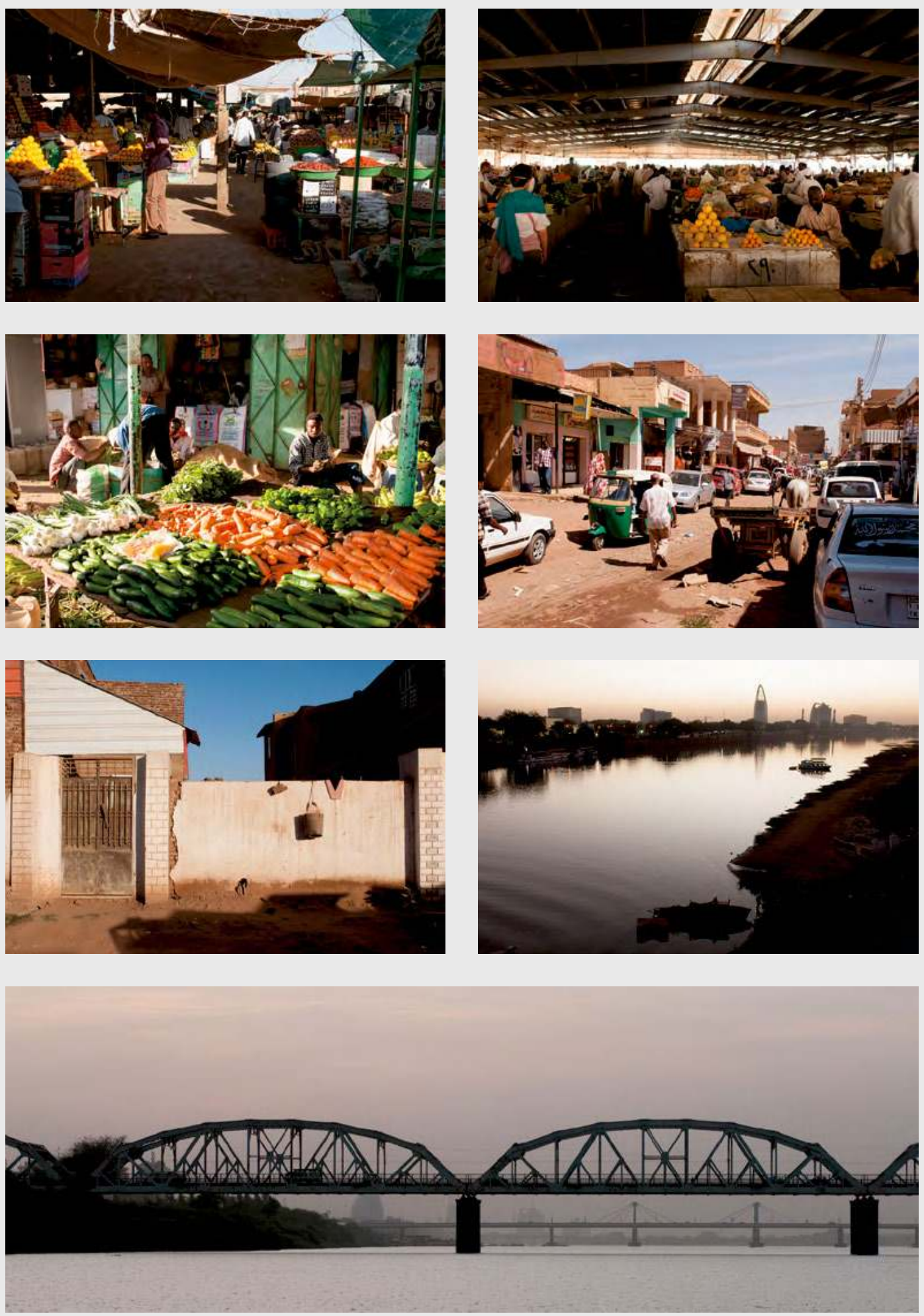

Les différents souks traditionnels d'Omdurman (alimentation, antiquités, bois...) et l'urbanisation de Khartoum. 

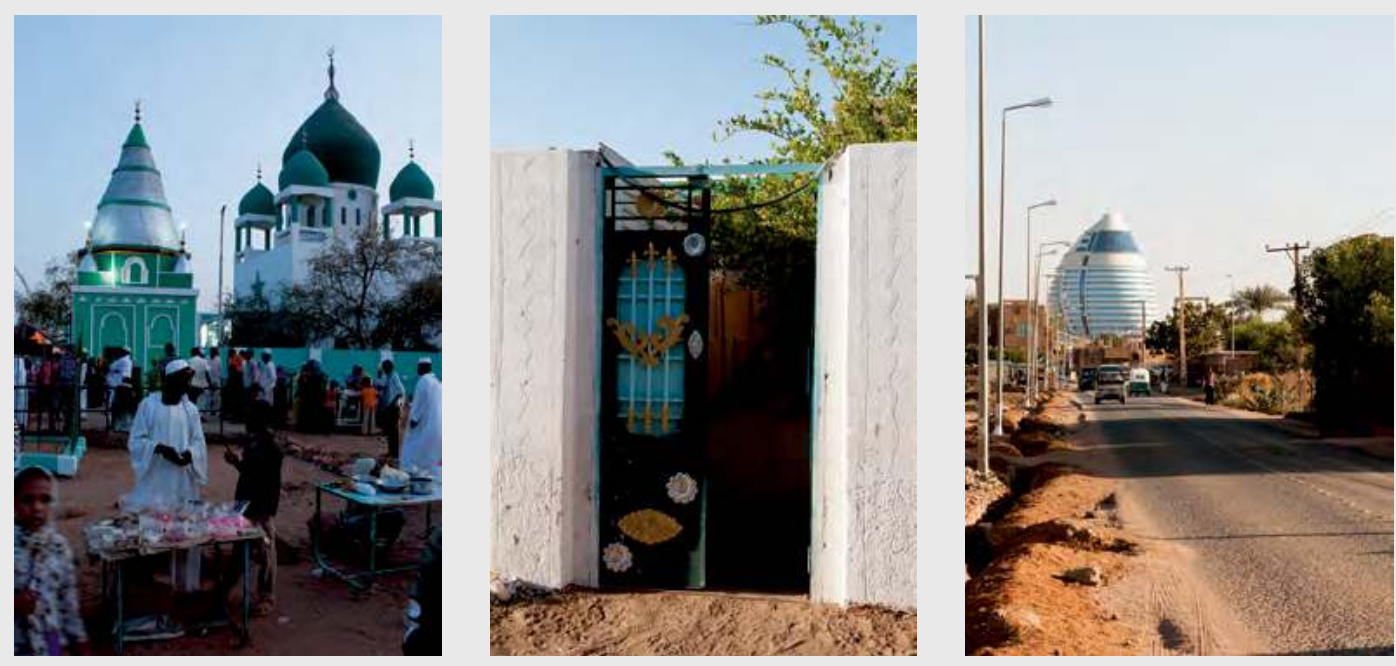

le Soudan
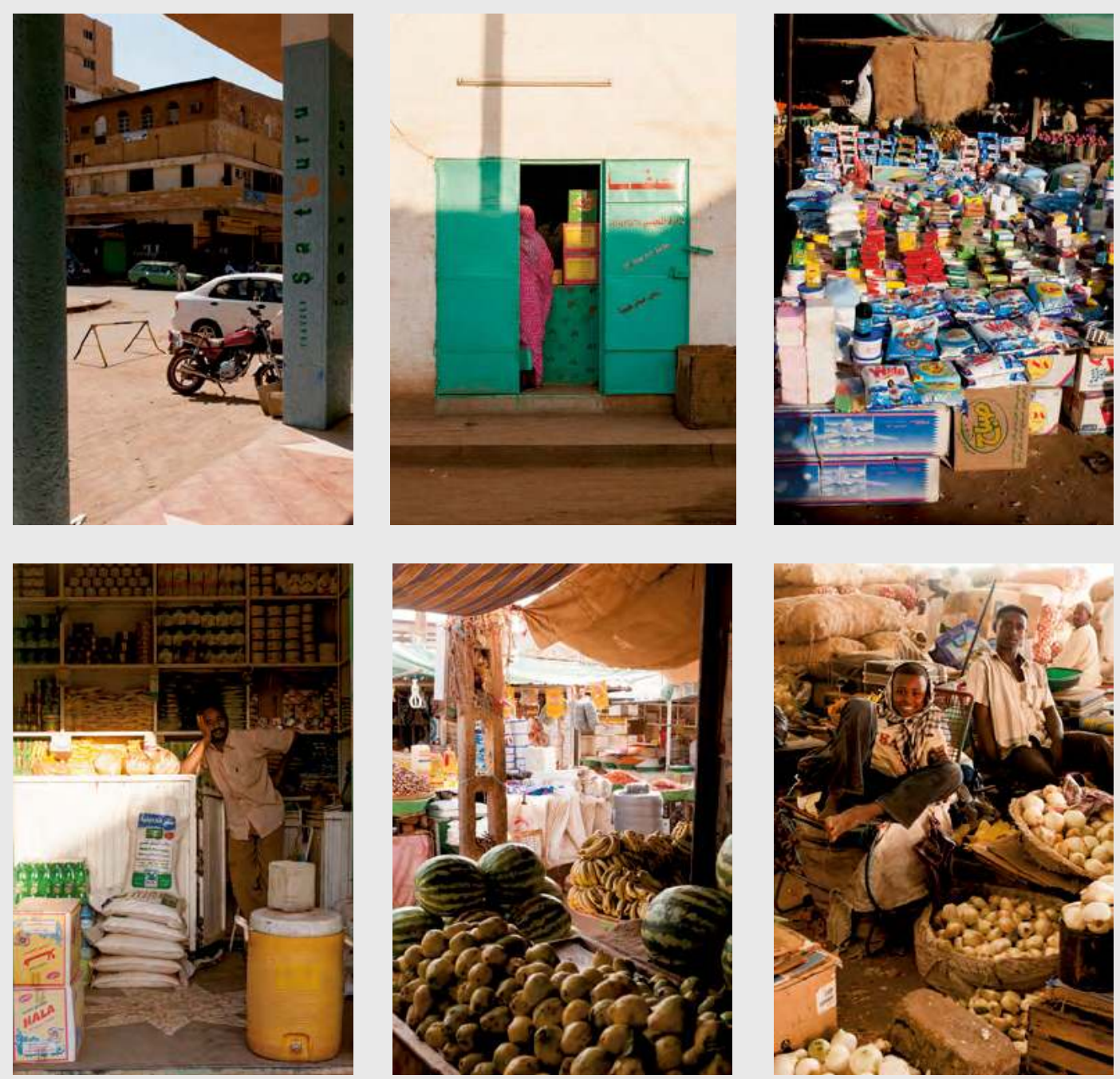


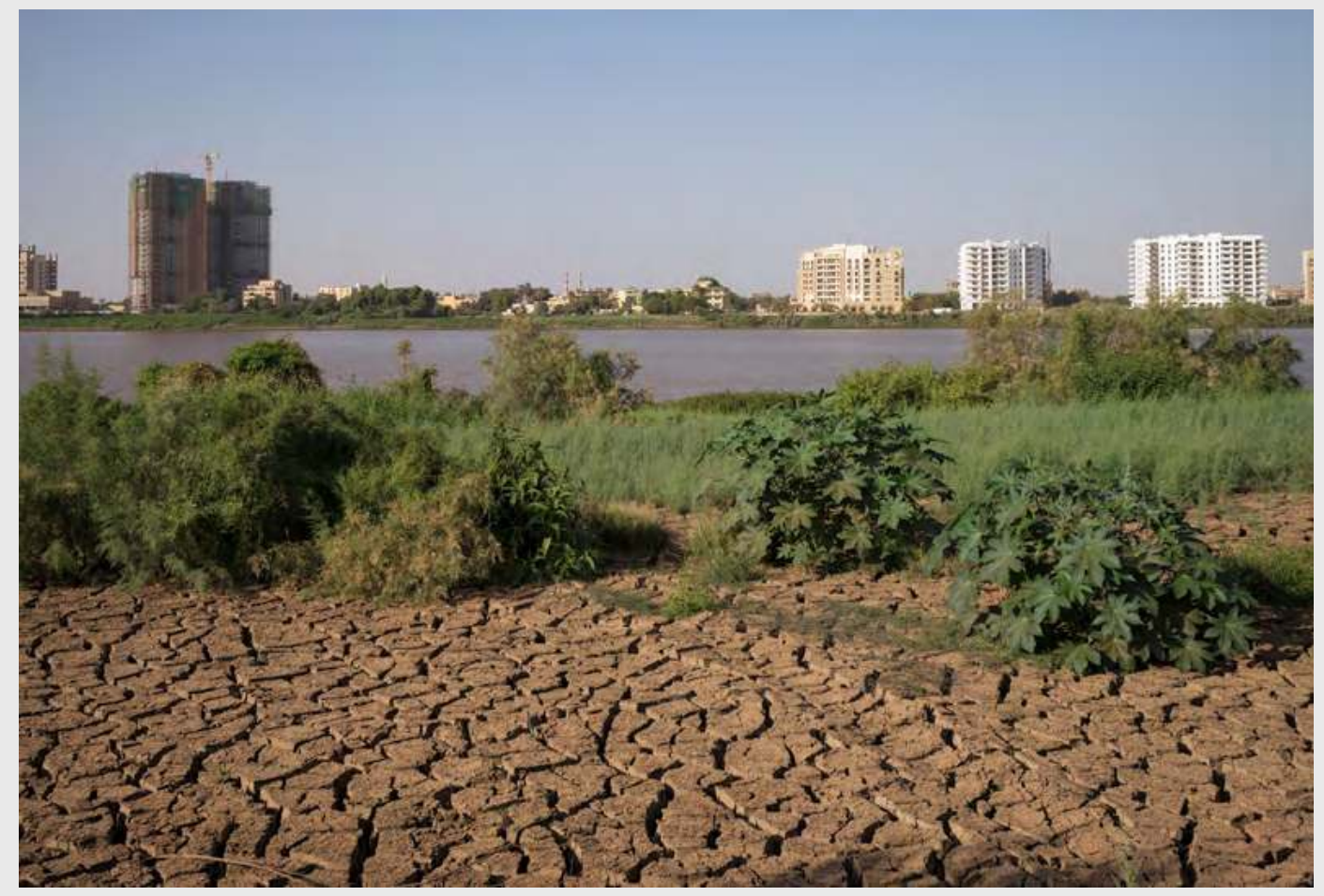

Les bords du Nil à Khartoum, en 2016 .

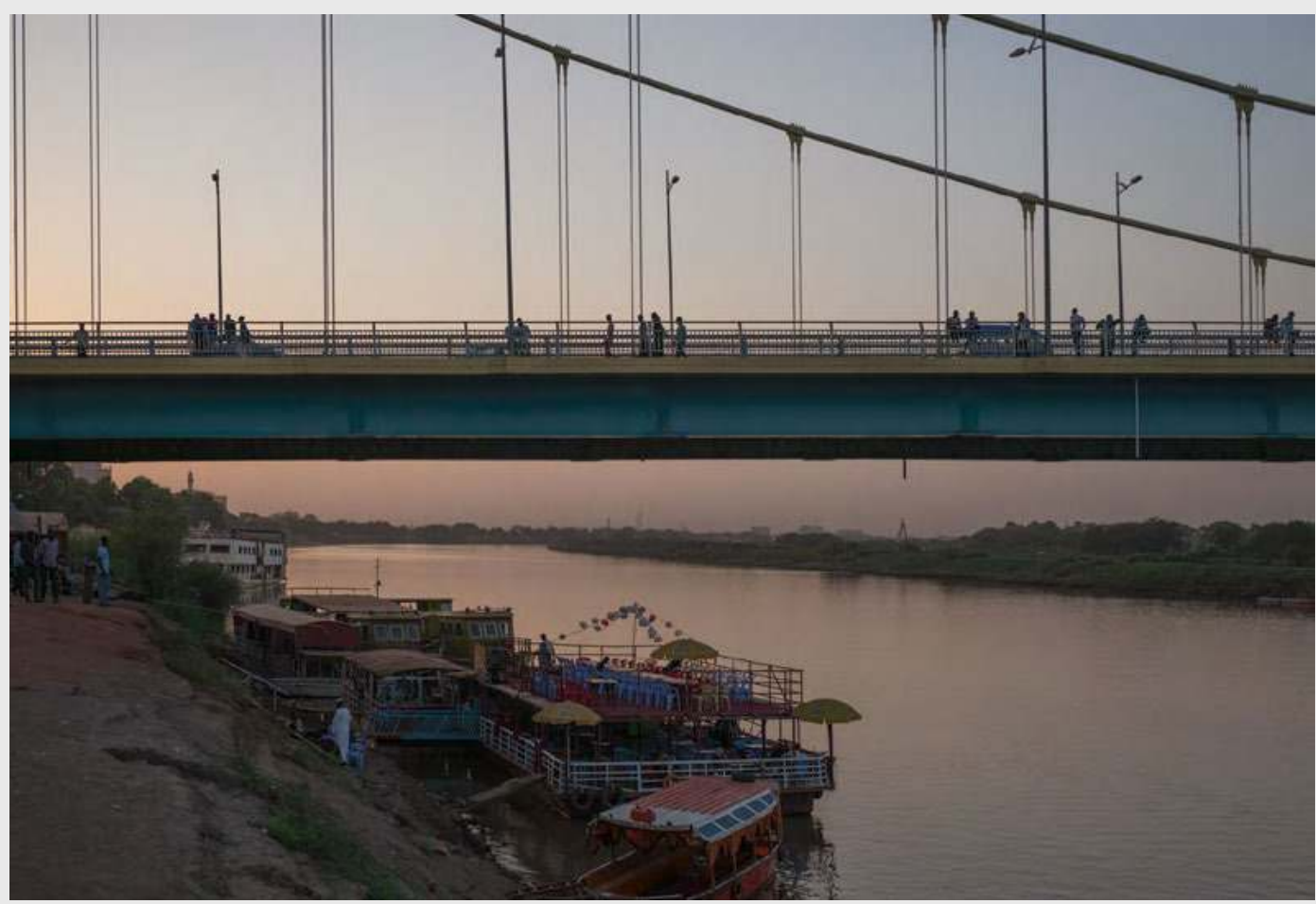


qui ont accueilli successivement les victimes de la sécheresse des années 1980 et ceux du conflit Nord-Sud des années 1990. Il y a un vrai contraste entre les projets de développement immobilier pharaoniques et les difficultés à répondre aux demandes de logement. Les rives du Nil, elles, en raison des crues annuelles, sont toujours dans leur état naturel, et des cultures saisonnières (oignons, sorgho, tomates...) sont pratiquées dès que commence la décrue.

\section{comment se déplacer}

le Soudan

792

aujourd'hui

L'étendue du pays rend difficile le développement du réseau de transport. Il existe quelques voies ferrées reliant les grandes villes à Khartoum, ainsi que des routes goudronnées, souvent en mauvais état. Mais où que vous vouliez aller, au Soudan ou à Khartoum, les solutions pour vous déplacer sont infiniment variées. Plusieurs lignes d'autobus traversent le pays et relient les principales villes - les plus récents sont relativement confortables. Plus rapides sont les boksi, pick-up de marque Toyota: seuls deux passagers prennent place à côté du chauffeur, les autres se serrent sur la plateforme à l'air libre et "profitent» du sable, du soleil et du vent. Vous pouvez aussi vous hisser sur le haut d'un camion.

À Khartoum même, le gouvernorat a mis en place depuis quelques années des autobus urbains pour compléter la myriade de minibus privés qui sillonnent la ville. Les taxis sont nombreux, les jaunes sont anciens et souvent en mauvais état; les Damas ou amjad (microbus), moins onéreux, peuvent être utilisés collectivement ou individuellement; quant aux rickshaws (ou tuk-tuk) venus d'Asie, ils permettent de circuler rapidement en centre-ville et de se faufiler dans les embouteillages... mais à vos risques et périls, et, en théorie, ils ne sont pas autorisés sur les grands axes.

\section{Khartoum en 2030...}

Des projets prestigieux laissent présager un Khartoum inspiré de Dubaï. Un bureau d'études a élaboré pour le gouvernorat les principaux projets pour ce Khartoum du futur. La construction d'un quartier d'affaires à la confluence du Nil, entre Khartoum et Omdurman, est déjà lancée: l'hôtel Burj el-Fateh (devenu Corinthia), construit par les Libyens et présent sur tous les dépliants touristiques est l'illustration des goûts architecturaux des pays du Golfe; dans le même quartier se trouvent la banque centrale et quelques tours, sièges de groupes pétroliers; une nouvelle corniche située sur l'avenue du Nil vient de voir le jour et sert déjà de lieu de promenade aux Soudanais qui, le soir, viennent s'y rafrâchir, prendre le thé, jouer 
aux cartes en famille et entre amis; ce quartier devrait également devenir un centre culturel et artistique; une corniche sur le Nil Blanc pourrait être créée sur le même modèle que la précédente.

Un centre commercial et un marché couvert devraient remplacer l'actuel aéroport, lorsque le nouveau, qui est à l'étude, sera construit plus au sud. Une rocade au sud sur le Nil Bleu et deux nouveaux ponts, pour rejoindre l'île de Tuti, devraient rendre la circulation plus fluide. La zone industrielle sera éloignée du centre-ville actuel qui pourrait devenir un quartier d'affaires et administratif. Bien sûr, l'étude propose l'amélioration des transports ainsi que la construction d'habitations à bas coût et à prix modéré. Les loisirs et le tourisme ne sont pas oubliés, puisque l'île de Tuti devrait abriter des villas de vacances. Des espaces verts devraient longer la voie de chemin de fer. La vieille ville d'Omdurman pourrait être dotée d'un grand marché couvert moderne mais conserver la partie qui abrite les artisans.

Ce sont quelques-uns des projets censés faire de Khartoum une capitale moderne. Tout cela est bien sûr conditionné par la situation économique: depuis la séparation d'avec le Sud et en l'absence des revenus pétroliers, tous les projets restent plus ou moins en attente

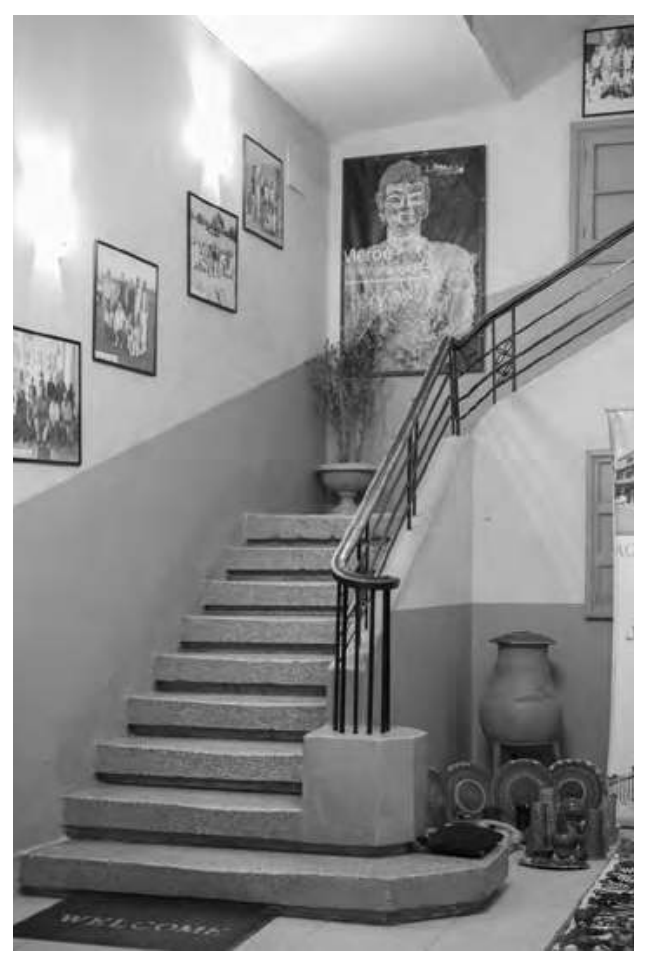

